\title{
AN EASY EXAMPLE OF A 0-SPACE NOT ALMOST RIMCOMPACT
}

\author{
BEVERLY DIAMOND
}

(Communicated by Dennis Burke)

\begin{abstract}
We construct an easy example of a space $X$ which is not almost rimcompact but for which $\beta X \backslash X$ is strongly 0-dimensional.
\end{abstract}

Recall that a space $X$ is rimcompact if $X$ possesses a base of open sets with compact boundaries, and almost rimcompact if $X$ has a conpactification $K X$ in which points of $K X \backslash X$ have a base of open sets of $K X$ whose boundaries lie in $X$. Any rimcompact space is almost rimcompact (see, for example, VI, example 30, of [Is]); and any almost rimcompact space is clearly a 0 -space, that is, has a compactification with 0 -dimensional remainder. In VI, example 7 , of [Is], Isbell indicates a construction involving the product of a 0 -dimensional space and an ordinal space which yields an almost rimcompact space that is not rimcompact. Using different techniques, he goes on to produce a much more complicated example of a space $X$ which is not rimcompact (or even almost rimcompact), but has a compactification $K X$ with $K X \backslash X$ strongly 0 -dimensional (that is, $\operatorname{dim}(K X \backslash X)=0)$. We show that a straightforward use of the easier construction can be used to produce a space $X$ having the latter properties and with $K X=\beta X$.

Let $\mathscr{R}$ denote a maximal almost disjoint collection of infinite subsets of the natural numbers $N$. The space $N \cup \mathscr{R}$ has the topology described in $5 \mathrm{I}$ of [GJ]; each point of $N$ is isolated, and $\lambda \in \mathscr{R}$ has an open base $\{\{\lambda\} \cup(\lambda \backslash F): F$ is a finite subset of $N\}$. The space $N \cup \mathscr{R}$ is locally compact, pseudocompact, and 0-dimensional. According to 2.1 and the concluding remarks of [Te], given any first-countable separable compact Hausdorff space $T$, there is a family $\mathscr{R}$ so that $\beta(N \cup \mathscr{R}) \backslash(N \cup \mathscr{R})$ is homeomorphic to $T$. If we choose $T$ to be the unit interval $I$, then $N \cup \mathscr{R}$ is not strongly 0 -dimensional, and $N \cup \mathscr{R} \cup\{0\}$ is totally disconnected but not 0 -dimensional. The subspace $\mathscr{R}$ is discrete, so that the space $\mathscr{R} \cup\{0\}$ has only one nonisolated point; hence $\operatorname{dim}(\mathscr{R} \cup\{0\})=0$. The point 0 does not have an open neighborhood $U$ in $\beta(N \cup \mathscr{R})$ with $\frac{1}{2} \notin U$ and $\mathrm{bd}_{\beta(N \cup \mathscr{R})} U \cap \mathscr{R}=\varnothing$, for if such a $U$ exists, then $\left[\mathrm{bd}_{\beta(N \cup \mathscr{R})} U\right] \cap(N \cup \mathscr{R})=\varnothing$, since points of $N$ are isolated in $\beta(N \cup \mathscr{R})$.

Received by the editors February 1, 1989.

1980 Mathematics Subject Classification (1985 Revision). Primary 54D40, 54G20.

(C) 1990 American Mathematical Society $0002-9939 / 90 \$ 1.00+\$ .25$ per page 
That is, $U \cap(N \cup \mathscr{R})$ is open and closed in $N \cup \mathscr{R}$, and $c l_{\beta(N \cup \mathscr{R})}[U \cap(N \cup \mathscr{R})]$ disconnects $\beta(N \cup \mathscr{R}) \backslash(N \cup \mathscr{R})$, a contradiction.

Let $Y=\beta(N \cup \mathscr{R}) \times\left(\omega_{1}+1\right)$, and $X=Y \backslash\left[(\mathscr{R} \cup\{0\}) \times\left\{\omega_{1}\right\}\right]$. It follows from Theorems 1 and 4 of [Gl] and 6.7 of [GJ] that $\beta X=Y$. According to 2.8 of [Di], if $X$ is almost rimcompact and $\beta X \backslash X$ is 0-dimensional, points of $\beta X \backslash X$ have bases in $\beta X$ of open sets with boundaries in $X$. The point $\left(0, \omega_{1}\right)$ does not have such a base, since the intersections of such sets with $\beta(N \cup \mathscr{R}) \times\left\{\omega_{1}\right\}$ would constitute a base for $\left(0, \omega_{1}\right)$ in $\beta(N \cup \mathscr{R}) \times\left\{\omega_{1}\right\}$ which cannot exist. Then the space $X$ has the desired properties.

\section{ACKNOWLEDGMENT}

The author wishes to thank the American Association of University Women for financial support.

\section{REFERENCES}

[Di] B. Diamond, Almost rimcompact spaces, Topology Appl. 25 (1987), 81-91.

[GJ] L. Gillman and M. Jerison, Rings of continuous functions, Van Nostrand, New York, 1960.

[Gl] I. Glicksberg, Stone-Cech compactifications of products, Trans. Amer. Math. Soc. 90 (1959), 369-382.

[Is] J. R. Isbell, Uniform spaces, Math. Surveys Monogr. 12 (1962).

[Te] J. Terasawa, Spaces $N \cup \mathscr{R}$ and their dimensions, Topology Appl. 11 (1980), 93-102.

Department of Mathematics, College of Charleston, Charleston, South Carolina 